\title{
Thermal Preconditioning and Heat-Shock Protein 72 Preserve Synaptic Transmission during Thermal Stress
}

\author{
Jonathan D. Kelty, ${ }^{1}$ Peter A. Noseworthy, ${ }^{2}$ Martin E. Feder, ${ }^{1}$ R. Meldrum Robertson, ${ }^{2}$ and \\ Jan-Marino Ramirez ${ }^{1}$ \\ ${ }^{1}$ Department of Organismal Biology and Anatomy, The University of Chicago, Chicago, Illinois 60637, and 2Department of \\ Biology, Queen's University, Kingston, Ontario, Canada, K7L 3N6
}

As with other tissues, exposing the mammalian CNS to nonlethal heat stress (i.e., thermal preconditioning) increases levels of heat-shock proteins (Hsps) such as Hsp70 and enhances the viability of neurons under subsequent stress. Using a medullary slice preparation from a neonatal mouse, including the site of the neural network that generates respiratory rhythm (the preBötzinger complex), we show that thermal preconditioning has an additional fundamental effect, protection of synaptic function. Relative to $30^{\circ} \mathrm{C}$ baseline, initial thermal stress $\left(40^{\circ} \mathrm{C}\right)$ greatly increased the frequency of synaptic currents recorded without pharmacological manipulation by $\sim 17$-fold $(p<0.01$ ) and of miniature postsynaptic currents (mPSCs) elicited by GABA (20-fold) glutamate (10-fold), and glycine (36-fold). Ther- mal preconditioning $\left(15 \mathrm{~min}\right.$ at $\left.40^{\circ} \mathrm{C}\right)$ eliminated the increase in frequency of overall synaptic transmission during acute thermal stress and greatly attenuated the frequency increases of GABAergic, glutamatergic, and glycinergic mPSCs (for each, $p<0.05$ ). Moreover, without thermal preconditioning, incubation of slices in solution containing inducible Hsp70 (Hsp72) mimicked the effect of thermal preconditioning on the stressinduced release of neurotransmitter. That preconditioning and exogenous Hsp72 can affect and preserve normal physiological function has important therapeutic implications.

Key words: hyperthermia; heat shock; synaptic transmission; miniature postsynaptic current; GABA; glutamate; glycine
The discovery that preconditioning with mild stress protects neural tissue from severe stresses such as hyperthermia or hypoxia has yielded new ideas on how to mitigate brain damage by ischemia and stroke (Snider et al., 1998; Perrault and Menasche, 1999; Zhang et al., 2000). Among other mechanisms, preconditioning involves stress or heat-shock proteins (Hsps), including molecular chaperones that minimize aggregation of and/or promote the productive refolding of stress-damaged proteins (Bellmann et al., 1996; Sato et al., 1996; Kiang and Tsokos, 1998; Yenari et al., 1998; Feder and Hofmann, 1999; Yang and Lin, 1999; Ohtsuka and Suzuki, 2000; Rajdev et al., 2000). In the mammalian CNS, as in numerous other tissues and organisms, preconditioning can elicit expression of a primarily inducible member of the DnaK-70 kDa Hsp family (Hsp72 in mouse) that facilitates cell survival during subsequent stress (Amin et al., 1996; Kiang and Tsokos, 1998; Yenari et al., 1999; Ohtsuka and Suzuki, 2000).

Although preconditioning and Hsp72 clearly enhance the capacity of neurons to survive stress, the fundamental issue of whether either can protect or modulate communication between stressed neurons is unresolved. In the mammalian CNS, constitutively expressed molecular chaperones, including Hsp70s, associate with proteins at presynaptic and postsynaptic sites and may be important for normal synaptic function (DeLuca-Flaherty et

\footnotetext{
Received Aug. 6, 2001; revised Oct. 4, 2001; accepted Oct. 9, 2001.

This research was funded by grants from the National Institutes of Health (NIH) (J.-M.R.) and the National Science Foundation (M.E.F.). J.D.K. was supported by a postdoctoral training grant from NIH. We thank Mark Musch for technical assistance with Western blotting.

Correspondence should be addressed to Dr. Jonathan D. Kelty, Department of Organismal Biology and Anatomy, The University of Chicago, 1027 East 57th Street, Chicago, IL 60637. E-mail: j-kelty@uchicago.edu.

Copyright (C) 2002 Society for Neuroscience $\quad 0270-6474 / 02 / 220001-\bullet \$ 15.00 / 0$
}

al., 1990; Stantchev, 1994; Jiang et al., 2000; Ohtsuka and Suzuki, 2000; Newmyer and Schmid, 2001). Furthermore, preconditioning Drosophila larvae with brief exposure to $37^{\circ} \mathrm{C}$ induces $\mathrm{Hsp} 70$ expression and preserves synaptic transmission during subsequent exposure to high temperature (Karunanithi et al., 1999). Thus, we first examined whether thermal preconditioning affected synaptic transmission between thermally stressed neurons in a mouse brainstem slice preparation. Although, our emphasis is on examining the effects on synaptic transmission in general, we chose this preparation because it includes the pre-Bötzinger complex (PBC), the location of the neural network controlling normal breathing rhythm, gasping, and sighs (Ramirez et al., 1998; Lieske et al., 2000) (Fig. 1a). Mechanisms that protect against stress-induced changes in synaptic transmission within this preparation may have implications for autonomic CNS function. To determine how these changes relate specifically to respiratory function in particular (e.g., resting membrane potential, ionic currents, bursting properties, and synaptic currents), future studies will investigate the effects on functionally identified respiratory neurons.

This article is published in The Journal of Neuroscience, Rapid Communications Section, which publishes brief, peerreviewed papers online, not in print. Rapid Communications are posted online approximately one month earlier than they would appear if printed. They are listed in the Table of Contents of the next open issue of JNeurosci. Cite this article as: JNeurosci, 2002, 22:RC193 (1-6). The publication date is the date of posting online at www.jneurosci.org.

http://www.jneurosci.org/cgi/content/full/5937 
a

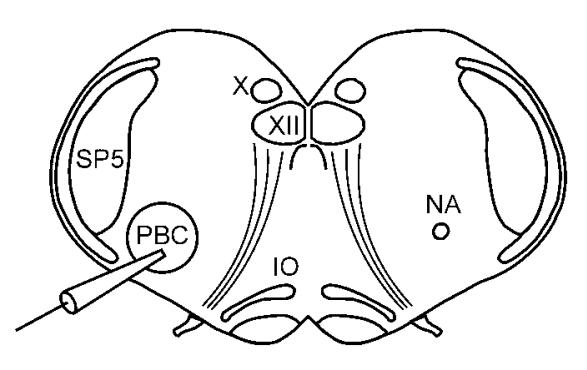

Figure 1. Thermal stress $\left(40^{\circ} \mathrm{C}\right)$ and preconditioning affected the frequency of combined spontaneous and action potential-evoked neurotransmitter release. $a$, Brainstem slice containing the PBC, the hypoglossal nucleus $(X I I)$, the inferior olive $(I O)$, the spinal trigeminal nucleus $(S P 5)$, the nucleus ambiguous $(N A)$, and the vagus motor nucleus $(X)$ (Ramirez et al., 1996, 1998; Lieske et al., 2000). b, Thermal preconditioning $1 \mathrm{hr}$ before thermal stress $(\square, n=6)$ mitigated the frequency increase observed during thermal stress in naive neurons $(\diamond, n=4 ; p<$ $0.05)$. Each point represents the mean frequency of postsynaptic events occurring during sequential 1 min recordings. $c$, An example of a whole-cell recording of the effects of thermal stress on frequency of synaptic activity in a neuron from a naive slice (left) and a preconditioned slice (right). Calibration: $200 \mathrm{pA}, 200 \mathrm{msec}$. $V_{\mathrm{h}}=-60 \mathrm{mV}$.

Without Preconditioning b
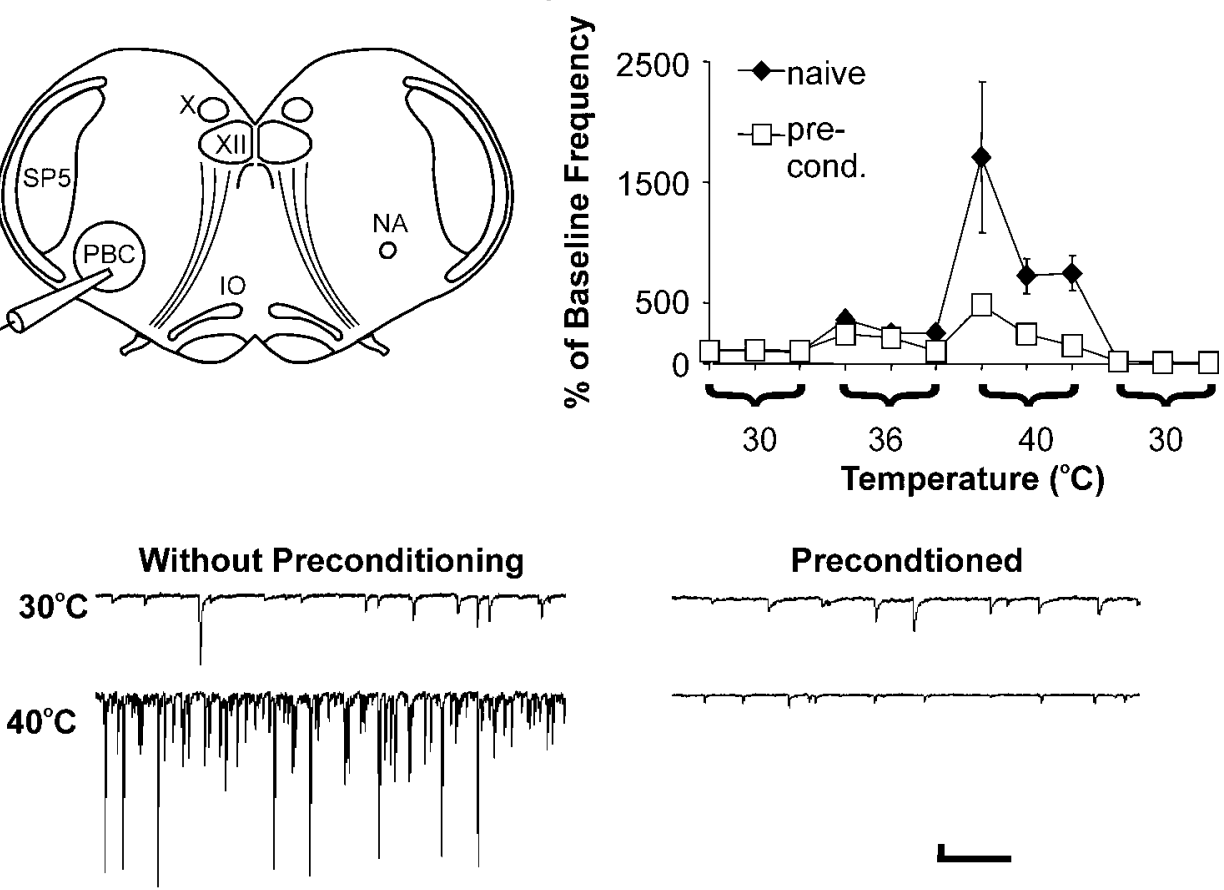

\section{Precondtioned}

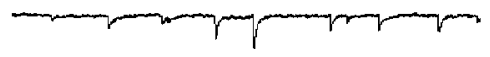

Neurons and other cells can take up Hsc70 (i.e., a constitutively expressed Hsp70 family member) and Hsp72 from the external medium, and supplying exogenous Hsps to these cells increases their survival of stress (Guzhova et al., 1998; Yu et al., 2001). Thus, we also determined whether incubating our slice preparation in a solution containing recombinant Hsp72 could modulate synaptic transmission during thermal stress. Our data demonstrate for the first time that both thermal preconditioning and incubation in an Hsp72 solution protect synaptic transmission during thermal stress. These findings open a new avenue of research on how stress and stress proteins affect neuronal function.

\section{MATERIALS AND METHODS}

General methods. Functionally intact transverse slices of mouse brainstem (medulla) were obtained from 2- to 8-d-old male and female CD-1 mice that were deeply anesthetized with ether and decapitated at the $\mathrm{C} 3 / \mathrm{C} 4$ spinal level (Fig. 1a) (Ramirez et al., 1996). The brainstem was isolated in ice-cold carbogen-equilibrated $\left(95 \% \mathrm{O}_{2}\right.$ and $\left.5 \% \mathrm{CO}_{2}\right)$ artificial CSF (aCSF) (in mM: $118 \mathrm{NaCl}, 3 \mathrm{KCl}, 1.5 \mathrm{CaCl}_{2}, 1 \mathrm{MgCl}_{2} * 6 \mathrm{H}_{2} \mathrm{O}, 25$ $\mathrm{NaHCO}_{3}, 1 \mathrm{NaH}_{2} \mathrm{PO}_{4}$, and 30 D-glucose, $\mathrm{pH}$ 7.4) and mounted in a vibrating microtome; approximately $250 \mu \mathrm{m}$ slices were removed from the rostral end until the PBC was revealed. At this level, a 500- $\mu \mathrm{M}$-thick slice was removed and equilibrated at $30^{\circ} \mathrm{C}$ in circulating carbogenequilibrated aCSF for $30 \mathrm{~min}$ before subsequent treatment.

Recordings. Using conventional whole-cell patch clamp, synaptic currents were recorded from random neurons in the region containing the PBC (i.e., in portions of the slice ventral to the level of the nucleus ambiguous, medial to the spinal trigeminal nucleus, and lateral to the hypoglossal nerve tract) (Fig. 1a). This region is capable of generating spontaneously respiratory activity in the presence of $8 \mathrm{~mm}$ potassium. However, in the present study, we used the more physiological concentration of $3 \mathrm{~mm}$. Under these conditions rhythmic activity ceases, thereby preventing us from identifying whether a neuron is respiratory. Unpolished electrodes were fabricated from thick-walled borosilicate glass (GC 150-10; Warner Instruments, Hamden, CT) and filled with (in mM): 140 $\mathrm{KCl}$ [or potassium gluconate when recording glutamatergic miniature postsynaptic currents (mPSCs)], $1 \mathrm{CaCl}_{2}, 2 \mathrm{MgCl}_{2}, 4 \mathrm{Na}_{2} \mathrm{ATP}, 10$ EGTA, and 10 HEPES, pH 7.2. An on-cell patch configuration was established under visual control using infra-red Normarski optics $(40 \times)$. A whole-cell configuration was established by applying repetitive pulses of negative pressure until the cell membrane within the tip of the electrode ruptured. Transmembrane currents were recorded using an Axopatch 1-D amplifier (Axon Instruments, Union City, CA) and recorded on the hard disk of a personal computer using pClamp 8 software and a Digidata 1200B data acquisition board (Axon Instruments). Before recording any data from a cell, transient currents attributable to electrode and cell resistance and capacitance were nullified as much as possible and serial resistance was $80 \%$ compensated.

Frequencies of combined spontaneous and action potential-evoked synaptic events were determined from three $1 \mathrm{~min}$ recordings at each temperature (below). To record mPSCs, action potential production was blocked with $1 \mu \mathrm{M}$ tetrodotoxin (TTX). Additional pharmacological manipulations isolated transmission via GABA [DNQX $(20 \mu \mathrm{M})$ and strychnine hydrochloride $(1 \mu \mathrm{M})$ ], glutamate [strychnine $(1 \mu \mathrm{M})$ and bicuculline $(20 \mu \mathrm{M})$ ], and glycine [DNQX $(20 \mu \mathrm{M})$ and bicuculline $(20$ $\mu \mathrm{M})]$. Beginning 5-10 min after drug addition, mPSCs were recorded for 2 min each at each temperature (below).

Preconditioning, incubation treatments, and thermal stress. To test the effect of thermal preconditioning on synaptic function during thermal stress, slices were either (1) kept at $30^{\circ} \mathrm{C}$ for $1-2 \mathrm{hr}$ after initial equilibration (controls) or (2) heated to $40^{\circ} \mathrm{C}$ at $\sim 1^{\circ} \mathrm{C} / \mathrm{min}$, kept at this temperature for $15 \mathrm{~min}$, and then allowed to recover for 1 or $2 \mathrm{hr}$ at $30^{\circ} \mathrm{C}$. Neurons were patch-clamped 5-10 min before the end of recovery from thermal preconditioning or before the end of the sham incubation so that experimentation could be completed within $15 \mathrm{~min}$ after the end of recovery.

When testing the effect of treatment with exogenous Hsp72, medullary slices were incubated for $2 \mathrm{hr}$ in aCSF, aCSF containing $10 \mu \mathrm{g} / \mathrm{ml}$ BSA (69 $\mathrm{kDa}$ molecular mass), or aCSF containing $10 \mu \mathrm{g} / \mathrm{ml}$ recombinant Hsp72 (SPP-755; StressGen, San Diego, CA). Within $1 \mathrm{hr}$ after incubation, the effect of thermal stress on glutamatergic or glycinergic mPSCs was assessed in normal aCSF as stated below. If recordings were not completed within $1 \mathrm{hr}$ after incubation, the slice was discarded.

After recovery from the preconditioning treatment or after incubation treatment, slices were transferred to a recording chamber perfused with carbogen-equilibrated aCSF and synaptic currents were assessed at $30^{\circ} \mathrm{C}$. The slice was then successively heated at $\sim 1^{\circ} \mathrm{C} / \mathrm{min}$ to 36 and $40^{\circ} \mathrm{C}$. At each of these temperatures, synaptic transmission was assessed as quickly as possible $(<5 \mathrm{~min})$.

Immunoblots. Hearts and livers were harvested from anesthetized mice and treated in aCSF as described for the brainstem. Intestinal cell cultures (IEC18 cells) were sealed with Parafilm (Pichiney Plastic Packaging, Menasha, WI), placed in a shaking water bath at $42^{\circ} \mathrm{C}$ for 
a. GABA

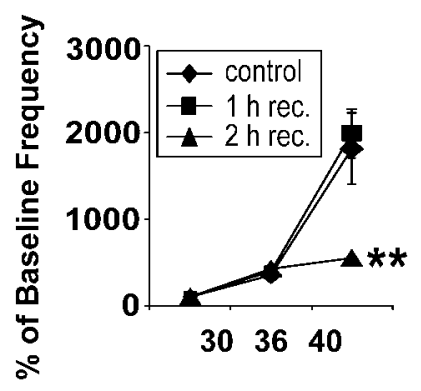

b. Glutamate

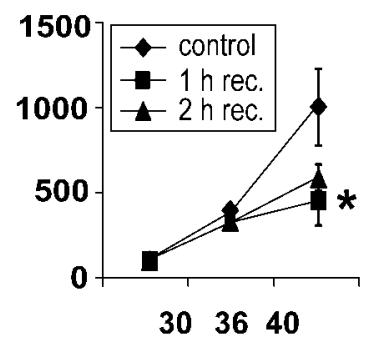

Temperature $\left({ }^{\circ} \mathrm{C}\right)$

\section{c. Glycine}

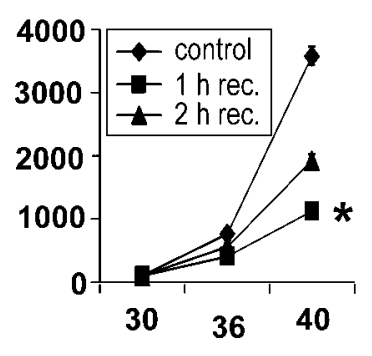

Figure 2. Effect of thermal preconditioning and thermal stress on the frequency of mPSCs elicited by GABA $(a)$, glutamate $(b)$, and glycine $(c)$. Only one set of data (i.e., from one neuron at 30,36 , and $40^{\circ} \mathrm{C}$ ) was taken from each slice. The seemingly inward glycinergic currents reflect the high chloride concentration of the intracellular solution used for the recordings. For all neurotransmitters, preconditioning 1-2 $\mathrm{hr}$ before thermal stress mitigated the increases in mPSC frequency seen during thermal stress in naive slices $\left({ }^{*} p<0.05 ;{ }^{* *} p<\right.$ $0.001)$. Sample sizes for $a-c$, respectively: $\bullet, 8,13$, and $7 ; \mathbf{\square}, 7,9$, and 8 ; $\boldsymbol{\Delta}, 9,8$, and 8. $d$, Sample recordings of glycinergic mPSCs during thermal stress without and after preconditioning. Calibration: 100 pA, 500 msec. $V_{\mathrm{h}}=-60 \mathrm{mV}$
$23 \mathrm{~min}$, and then allowed to recover for $2 \mathrm{hr}$ at $37^{\circ} \mathrm{C}$ in a $5 \% \mathrm{CO}_{2}$ incubator (Burress et al., 1997). After thermal treatment, brainstem slices and tissue samples were frozen in liquid nitrogen and stored at $-80^{\circ} \mathrm{C}$. Proteins were extracted by homogenizing samples in ice-cold PBS containing $2 \% \mathrm{w} / \mathrm{v}$ complete protease inhibitor (Boehringer Mannheim, Indianapolis, IN) and $50 \mathrm{U} / \mathrm{ml}$ DNase (Sigma, St. Louis, MO). Protein content was determined with a BCA assay (Pierce, Rockford, IL). Proteins were separated by electrophoresis of $10 \mu \mathrm{g}$ of total protein/lane of a $10 \%$ Tris-HCl SDS-polyacrylamide gel and were electroblotted onto a polyvinylidene difluoride membrane (Burress et al., 1997). After blocking with PBS containing $10 \%$ nonfat powdered milk, the membrane was washed successively in solutions containing primary antibody specific for inducible Hsp72 (SPA-810, diluted 1:1000; StressGen) or Hsc70 (SPA-815, diluted 1:2000; StressGen) as well as peroxidase-conjugated secondary antibody [goat antimouse IgG (115-036-062, diluted 1:10,000; Jackson ImmunoResearch, West Grove, PA) when detecting anti-Hsp72 primary or goat anti-rat IgG (112-035-003, diluted 1:10,000; Jackson ImmunoResearch, ) when detecting anti-Hsc70 primary]. Bound antigen was detected by chemiluminescence with the SuperSignal chemiluminescent substrate kit according to the manufacturer's instructions (34080; Pierce) and was recorded on x-ray film (Fuji, Tokyo, Japan).

Data analysis and statistical methods. We obtained a continuous 2 min recording for each neuron at each temperature step. From these recordings we measured the frequencies, amplitudes, and decay times of all postsynaptic events using MiniAnalysis 5.0 (SynaptoSoft Inc., Decatur, GA). Peak current amplitude was assessed as the difference between the amplitude of an event and the mean baseline current for the $4000 \mu \mathrm{sec}$ preceding the event. Decay time was assessed as the time at which mPSC amplitude decayed to $68 \%$ of the peak value. For a given recording, the values of each variable were averaged. Average values obtained from separate slices were then pooled and used to assess treatment effects. Pooled data were tested for normality with the Kolmogorov-Smirnov test using a software program (Sigma Stat; SPSS, Inc., Chicago, IL) and were then tested by ANOVA if distributed normally or by the Kruskal-Wallace test if non-normal. When merited, post hoc tests were used to compare individual treatment groups. Differences among treatments were considered significant at $p<0.05$.

\section{RESULTS}

\section{Effect of thermal preconditioning and thermal stress} on overall synaptic activity

In naive slices (i.e., without thermal preconditioning), the frequency of synaptic currents recorded without pharmacological manipulation responded biphasically to heating from 30 to $40^{\circ} \mathrm{C}$ (Fig. $1 b, c$ ). During heating from 30 to $36^{\circ} \mathrm{C}$ and at $36^{\circ} \mathrm{C}$ the frequency of overall transmission remained relatively constant, as shown quantitatively in Figure $1 b$. In contrast, during the first minute at $40^{\circ} \mathrm{C}$, the frequency increased by 17 -fold $(p<0.01)$. The frequency of transmission then decreased but remained elevated 7- to 7.5 -fold above $30^{\circ} \mathrm{C}$ levels during the next $2 \mathrm{~min}$. Thermal preconditioning $\left(15 \mathrm{~min}\right.$ at $\left.40^{\circ} \mathrm{C}\right) 1 \mathrm{hr}$ before thermal stress attenuated the heating-induced frequency increase, maintaining rates near baseline (Fig. 1b).

\section{Effects of thermal stress and preconditioning on miniature postsynaptic currents}

To determine whether thermal stress and preconditioning affected spontaneous neurotransmitter release, action potentials were blocked with $1 \mu \mathrm{M}$ TTX. Additional pharmacological manipulations isolated GABAergic, glutamatergic, and glycinergic mPSCs. In naive slices, exposure to $40^{\circ} \mathrm{C}$ increased the frequency of mPSCs elicited by all three neurotransmitters by at least 10 -fold (Fig. 2). Preconditioning at $40^{\circ} \mathrm{C}$ mitigated this increase, resulting in $\mathrm{mPSC}$ frequencies as low as threefold above baseline. This attenuation was evident by $1 \mathrm{hr}$ after preconditioning for glutamatergic and glycinergic mPSCs but was not apparent until $2 \mathrm{hr}$ after preconditioning for those elicited by GABA.

We subsequently analyzed the amplitude and decay of GABAergic, glutamatergic, and glycinergic mPSCs. Exposure to $40^{\circ} \mathrm{C}$ had no consistent effect on mPSC amplitude. In naive slices, $40^{\circ} \mathrm{C}$ thermal stress increased the amplitude of glutamatergic 


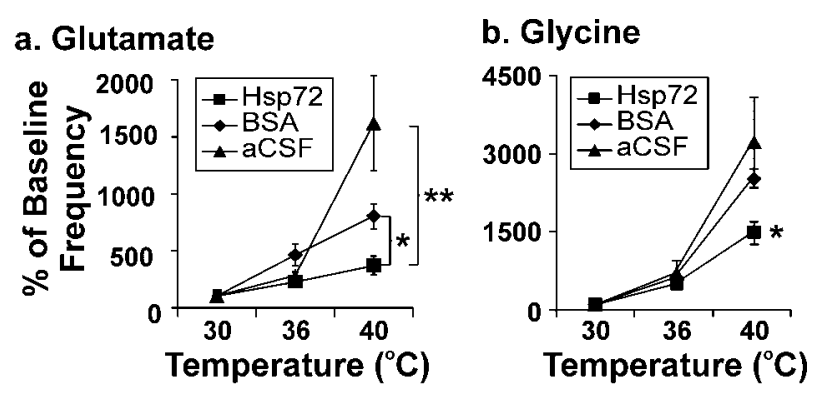

c. Example: Glycinergic MPSCs
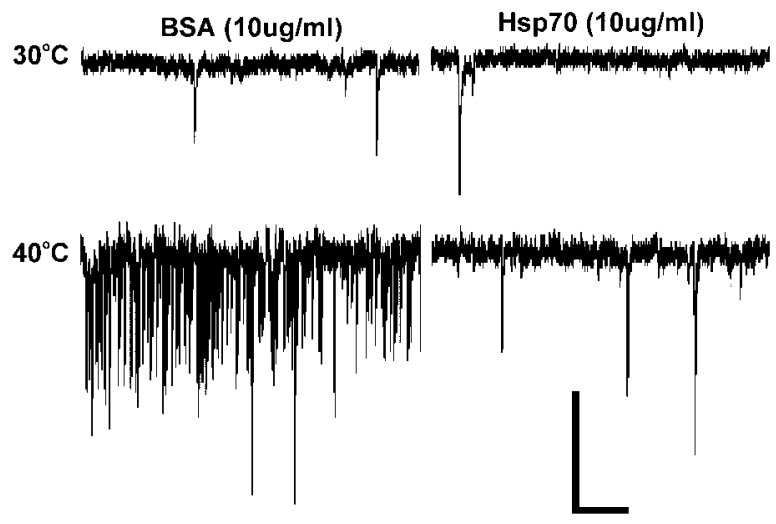

Figure 3. Effects of $2 \mathrm{hr}$ of incubation in aCSF, $10 \mu \mathrm{g} / \mathrm{ml}$ BSA in aCSF, or $10 \mu \mathrm{g} / \mathrm{ml} \mathrm{recombinant} \mathrm{Hsp72} \mathrm{(SPP-755;} \mathrm{StressGen)} \mathrm{in} \mathrm{aCSF} \mathrm{on} \mathrm{the}$ frequency of glutamatergic and glycinergic mPSCs during $40^{\circ} \mathrm{C}$ thermal stress. Only one set of data (i.e., 2 min recordings of synaptic activity at 30 , 36 , and $40^{\circ} \mathrm{C}$ ) was taken from each slice. Relative to slices incubated in aCSF or BSA solution, incubation in Hsp72 solution significantly attenuated the increase in glutamatergic $(a)$ and glycinergic $(b, c)$ mPSC frequency during thermal stress. Sample sizes in $a: \mathbf{\square}, 6 ; \bullet, 6 ; \mathbf{\Delta}, 6$. Sample sizes in $b: \mathbf{\square}, 8 ; \bullet, 9 ; \boldsymbol{\Lambda}, 7 .{ }^{*} p<0.05 ;{ }^{* *} p<0.01$. Calibration: 100 pA, 200 msec. $V_{\mathrm{h}}=-60 \mathrm{mV}$

mPSCs by $10.5 \pm 2.6 \%(p=0.02)$ but did not affect that of GABAergic or glycinergic mPSCs $(n=7-9$ slices per treatment; $p>0.05$ for each comparison). Additionally, in naive slices, exposure to $40^{\circ} \mathrm{C}$ decreased the decay times of both GABA and glycine mPSCs [by $43 \pm 3.8 \%(p<0.001)$ and $40 \pm 6.5 \%(p<$ $0.05)$, respectively] but not those of glutamate mPSCs $(p>0.73)$. The effects of thermal stress on both mPSC amplitude and decay were unaffected by thermal preconditioning $(p>0.05$ for each neurotransmitter), suggesting that thermal preconditioning primarily modulates presynaptic release mechanisms.

\section{Effect of incubation in recombinant Hsp72 before thermal stress}

Adding recombinant Hsp72 to the incubation medium mimicked the effects of thermal preconditioning on mPSCs elicited by both glutamate and glycine (Fig. 3). The effect of exogenous Hsp72 on GABAergic mPSCs was not examined. For slices incubated in aCSF only and in aCSF plus $\mathrm{BSA}, 40^{\circ} \mathrm{C}$ thermal stress increased the frequency of both glutamatergic and glycinergic mPSCs (by 12-fold and 32-fold, respectively, for aCSF). Although exposure to $40^{\circ} \mathrm{C}$ also increased the frequencies of glutamatergic and glycinergic mPSCs in slices incubated in Hsp72 solution (by 3.7-fold and 15-fold, respectively), these increases were substantially less than for slices incubated in aCSF only or in BSA solution. For all treatments (aCSF, BSA, and Hsp72), thermal stress did not affect the amplitude of either glutamatergic or glycinergic mPSCs $(p>$
$0.05)$ but decreased their decay times by $15-30 \%$ for glutamate $(p<0.05$ for each) and 30-50\% for glycine $(p<0.02)$. For both neurotransmitters, the effects of thermal stress on the amplitude and decay times of mPSCs from slices incubated in Hsp72 solution were indistinguishable from those of cells in control slices $(p>0.05)$. Thus, as with thermal preconditioning, these data indicate that incubation in Hsp72 modulated the effects of thermal stress on presynaptic release mechanisms without substantially affecting the postsynaptic response to neurotransmitter.

\section{Characterization of the heat-shock response of the medullary slice}

We used immunoblots to determine whether increases in Hsp72 and Hsc70 levels are part of the normal response of cells in the medullary slice to thermal stress. Slices used for this study were not used for electrophysiology. Using the immunoblotting technique, we were able to detect as little as $1 \mathrm{ng}$ of Hsp72 (Fig. 4d). Neither Hsp72 nor Hsc70 levels changed during the preconditioning regimen of the present study (Fig. 4a,c). However, more prolonged or severe heat shock and/or prolonged recovery increased Hsp72 levels in medullary slices (Fig. 4b).

\section{DISCUSSION}

Most previous work on protection against the deleterious impact of stresses (including hypoxia, ischemia, and hyperthermia) emphasized their consequences in terms of cell survival. We show that these consequences also extend to synaptic function, which may have a much lower threshold for damage than cell viability. Moreover, thermal preconditioning protects synaptic function just as it does cell viability. Additionally, we demonstrate that incubation of the medullary slice preparation in a solution containing recombinant $\mathrm{Hsp} 72$ protects synaptic function in much the same manner as thermal preconditioning.

For each neurotransmitter examined, thermal preconditioning mitigated the increase in mPSC frequency elicited by initial exposure to acute thermal stress. These data suggest both that thermal stress affects mechanisms controlling neurotransmitter release at the presynaptic terminal and, more importantly, that thermal preconditioning modulates these mechanisms. These data are consistent with findings for the neuromuscular junction of Drosophila melanogasater (Karunanithi et al., 1999) and, to our knowledge, are the first to demonstrate that thermal preconditioning modulates synaptic transmission in the mammalian CNS.

Using immunoblotting that detected as little as $1 \mathrm{ng}$ of Hsp72, we found that thermal preconditioning occurred without inducing detectable endogenous Hsp72. These data are in contrast to those from Drosophila (Karunanithi et al., 1999) and suggest one or more Hsp72-independent mechanisms, potentially involving other Hsps or non-Hsp-related processes (Kiang and Tsokos, 1998; Feder and Hofmann, 1999; Ohtsuka and Suzuki, 2000). Indeed, thermal stress induces expression of Hsp27 and Hsp32 at synaptic sites in the cerebellum (Bechtold and Brown, 2000). An open question is whether different preconditioning regimens, which cause physiological changes in Hsp72 levels, would cause attenuation of stress-induced increases in mPSC frequency similar to those observed after incubation in recombinant Hsp72 or thermal preconditioning. Such a finding would be consistent with numerous demonstrations that Hsp72 enhances cell survival throughout the CNS during diverse stress regimens (Amin et al., 1996; Sato et al., 1996; Yang and Lin, 1999; Ohtsuka and Suzuki, 2000; Rajdev et al., 2000).

As with thermal preconditioning, incubation of medullary 
a

$$
\begin{aligned}
& \text { Conditions Used in } \\
& \text { Electrophysiology }
\end{aligned}
$$

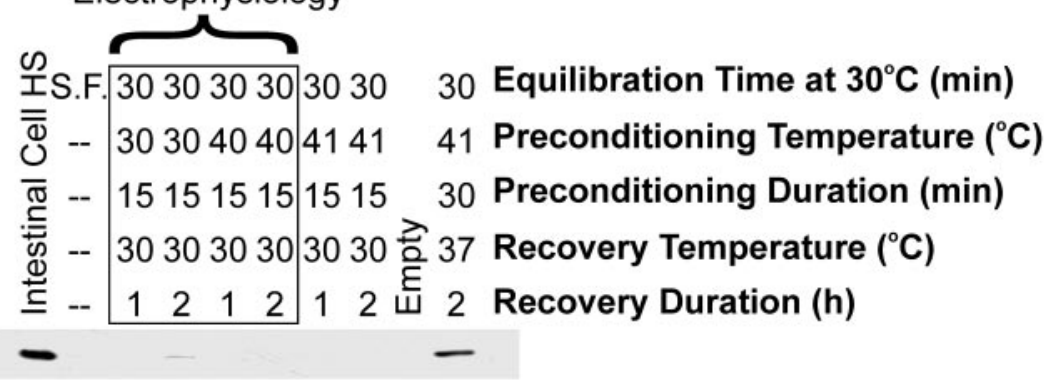

b

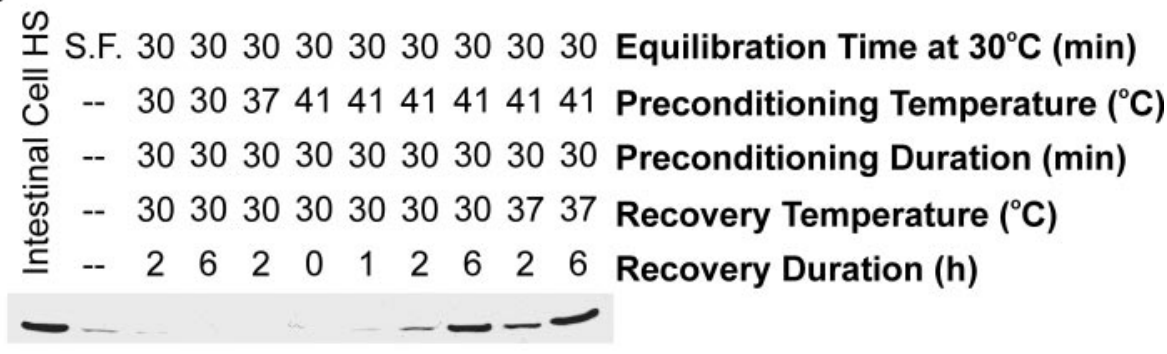

C

\section{Preconditioning}

Temperature:

$30^{\circ} \mathrm{C}$

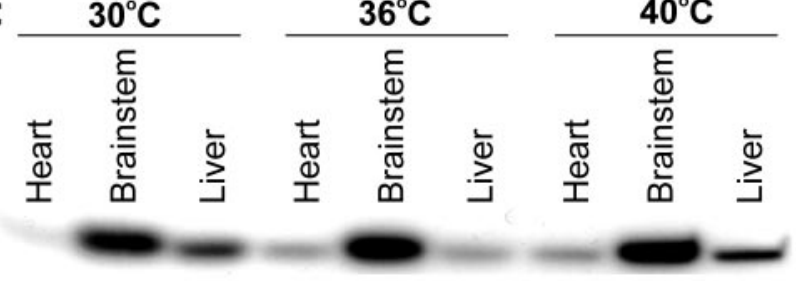

d

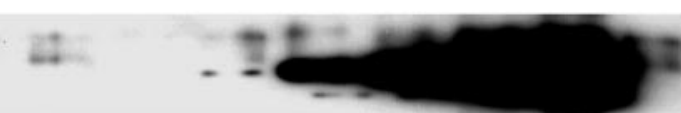

\section{Amount of Hsp70 loaded (ng):

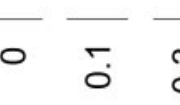$$
\text { m. }
$$

slices in a solution of recombinant Hsp72 attenuated increases in mPSC frequency during thermal stress. This effect could have occurred through numerous actions of exogenous Hsp72. Because this protein is taken up by neurons (Yu et al., 2001), it can directly interact with various intracellular proteins including cysteine string protein, a synaptic vesicle protein that interacts with $\mathrm{Hsp} 70$ family members and participates in the regulation of transmitter release (Jiang et al., 2000; Ohtsuka and Suzuki, 2000). Alternatively, Hsp72 could affect neuronal function by activating various cellular signaling pathways (Buzzard et al., 1998; Gabai et al., 2000).

Thermal stresses more severe and/or prolonged than the one we used to precondition slices induced substantial Hsp72, leading to two conclusions. First, increases in the level of Hsp72 are part of the normal cellular response to severe thermal stress in the medulla. Second, the low level of endogenous Hsp72 in our medullary slice preparation establishes that exogenous Hsp72 (Fig. 3) is sufficient for the mitigation of hyperthermic stress. Our experiments were performed in neonatal tissue, and it will be interesting to investigate whether similar or different adaptive mechanisms also occur in adult tissue and whether thermal tolerance undergoes a developmental change.

By attenuating massive increases in neurotransmitter release, both preconditioning and application of recombinant Hsp72 could protect against a major mechanism of stress-induced neuronal injury (Obrenovitch et al., 2000; Sattler and Tymianski, 2000). Application of exogenous Hsp72, which we show mimics the effect of thermal preconditioning, may elucidate the role of this protein and its cognate in synaptic transmission as well as in preservation of neuronal and synaptic function. Clinically, the capacity to manipulate and preserve normal physiological function via exogenous application of stress proteins suggests that such application could also be an important therapeutic tool.

\section{REFERENCES}

Amin V, Cumming DVE, Latchman DS (1996) Over-expression of heat shock protein 70 protects neuronal cells against both thermal and ischaemic stress but with different efficiencies. Neurosci Lett 206:45-48.

Bechtold DA, Brown IR (2000) Heat shock proteins Hsp27 and Hsp32 localize to synaptic sites in the rat cerebellum following hyperthermia. Brain Res Mol Brain Res 75:309-320. 
Bellmann K, Jaattela M, Wissing D, Burkart V, Kolb H (1996) Heat shock protein hsp70 overexpression confers resistance against nitric oxide. FEBS Lett 391:185-188.

Burress GC, Musch MW, Jurivich DA, Welk J, Chang EB (1997) Effects of mesalamine on the hsp72 stress response in rat IEC-18 intestinal epithelial cells. Gastroenterology 113:1474-1479.

Buzzard KA, Giaccia AJ, Killender M, Anderson RL (1998) Heat shock protein 72 modulates pathways of stress-induced apoptosis. J Biol Chem 273:17147-17153.

DeLuca-Flaherty C, McKay DB, Parham P, Hill BL (1990) Uncoating protein (hsc70) binds a conformationally labile domain of clathrin light chain LCa to stimulate ATP hydrolysis. Cell 62:875-887.

Feder ME, Hofmann GE (1999) Heat-shock proteins, molecular chaperones, and the stress response: evolutionary and ecological physiology. Annu Rev Physiol 61:243-282.

Gabai VL, Meriin AB, Yaglom JA, Wei JY, Mosser DD, Sherman MY (2000) Suppression of stress kinase JNK is involved in HSP72mediated protection of myogenic cells from transient energy deprivation. HSP72 alleviates the stress-induced inhibition of JNK dephosphorylation. J Biol Chem 275:38088-38094.

Guzhova IV, Arnholdt AC, Darieva ZA, Kinev AV, Lasunskaia EB, Nilsson K, Bozhkov VM, Voronin AP, Margulis BA' (1998) Effects of exogenous stress protein 70 on the functional properties of human promonocytes through binding to cell surface and internalization. Cell Stress Chaperones 3:67-77.

Jiang R, Gao B, Prasad K, Greene LE, Eisenberg E (2000) Hsc70 chaperones clathrin and primes it to interact with vesicle membranes. J Biol Chem 275:8439-8447.

Karunanithi S, Barclay JW, Robertson RM, Brown IR, Atwood HL (1999) Neuroprotection at Drosophila synapses conferred by prior heat shock. J Neurosci 19:4360-4369.

Kiang JG, Tsokos GC (1998) Heat shock protein $70 \mathrm{kDa}$ : molecular biology, biochemistry, and physiology. Pharmacol Ther 80:183-201.

Lieske SP, Thoby-Brisson M, Telgkamp P, Ramirez JM (2000) Reconfiguration of the neural network controlling multiple breathing patterns: eupnea, sighs, and gasps. Nat Neurosci 3:600-607.

Newmyer S, Schmid S (2001) Dominant-interfering hsc70 mutants disrupt multiple stages of the clathrin-coated vesicle cycle in vivo. J Cell Biol 152:607-620.

Obrenovitch T, Urenjak J, Zilkha E, Jay T (2000) Excitotoxicity in neurological disorders: the glutamate paradox. Int J Dev Neurosci 18:281-287.

Ohtsuka K, Suzuki T (2000) Roles of molecular chaperones in the nervous system. Brain Res Bull 53:141-146.

Perrault LP, Menasche P (1999) Preconditioning: can nature's shield be raised against surgical ischemic-reperfusion injury? Ann Thorac Surg 68:1988-1994.

Rajdev S, Hara K, Kokubo Y, Mestril R, Dillmann W, Weinstein PR, Sharp FR (2000) Mice overexpressing rat heat shock protein 70 are protected against cerebral infarction. Ann Neurol 47:782-791.

Ramirez JM, Quellmalz UJA, Richter DW (1996) Postnatal changes in the mammalian respiratory network as revealed by the transverse brainstem slice of mice. J Physiol (Lond) 491:799-812.

Ramirez J-M, Schwarzacher SW, Pierrefiche O, Olivera BM, Richter DW (1998) Selective lesioning of the cat pre-Botzinger complex in vivo eliminates breathing but not gasping. J Physiol (Lond) 507:895-907.

Sato K, Saito H, Matsuki N (1996) Hsp70 is essential to the neuroprotective effect of heat-shock. Brain Res 740:117-123.

Sattler R, Tymianski M (2000) Molecular mechanisms of calciumdependent excitotoxicity. J Mol Med 78:3-13.

Snider BJ, Lobner D, Yamada KA, Choi DW (1998) Conditioning heat stress reduces excitotoxic and apoptotic components of oxygen-glucose deprivation-induced neuronal death in vitro. J Neurochem 70:120-129.

Stantchev ZS (1994) Hsc70/uncoating ATPase activation: proposal of a new model. Med Hypotheses 43:138-140.

Yang Y-L, Lin M-T (1999) Heat shock protein expression protects against cerebral ischemia and monoamine overload in rat heatstroke. Am J Physiol 276:H1961-H1967.

Yenari MA, Fink SL, Sun GH, Chang LK, Patel MK, Kunis DM, Onley D, Ho DY, Sapolsky RM, Steinberg GK (1998) Gene therapy with HSP72 is neuroprotective in rat models of stroke and epilepsy. Ann Neurol 44:584-591.

Yenari MA, Giffard RG, Sapolsky RM, Steinberg GK (1999) The neuroprotective potential of heat shock protein 70 (HSP70). Mol Med Today 5:525-531.

Yu Q, Kent C, Tytell M (2001) Retinal uptake of intravitreally injected $\mathrm{Hsc} / \mathrm{Hsp} 70$ and its effect on susceptibility to light damage. Mol Vis 7:48-56.

Zhang P, Abraham VS, Kraft KR, Rabchevsky AG, Scheff SW, Swain JA (2000) Hyperthermic preconditioning protects against spinal cord ischemic injury. Ann Thorac Surg 70:1490-1495. 